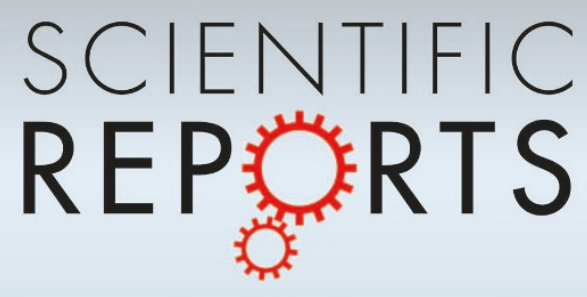

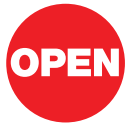

SUBJECT AREAS:

ELECTRONIC MATERIALS

AND DEVICES

APPLIED PHYSICS

MATERIALS PHYSICS

CONDENSED MATTER PHYSICS

Received

19 July 2012

Accepted

31 July 2012

Published

20 August 2012

Correspondence and requests for materials should be addressed to

A.L. (ALanzara@lbl. gov)

\section{Fermi velocity engineering in graphene by substrate modification}

\author{
Choongyu Hwang ', David A. Siegel ${ }^{1,2}$, Sung-Kwan $\mathrm{Mo}^{3}$, William Regan ${ }^{1,2}$, Ariel Ismach ${ }^{4}$, \\ Yuegang Zhang ${ }^{4}$, Alex Zettl ${ }^{1,2}$ \& Alessandra Lanzara ${ }^{1,2}$
}

\begin{abstract}
${ }^{1}$ Materials Sciences Division, Lawrence Berkeley National Laboratory, Berkeley, CA 94720, USA, ${ }^{2}$ Department of Physics, University of California, Berkeley CA 94720, USA, ${ }^{3}$ Advanced Light Source, Lawrence Berkeley National Laboratory, Berkeley, CA 94720, USA, ${ }^{4}$ The Molecular Foundry, Lawrence Berkley National Laboratory, Berkeley CA 94720, USA.
\end{abstract}

\begin{abstract}
The Fermi velocity, $v_{\mathrm{F}}$, is one of the key concepts in the study of a material, as it bears information on a variety of fundamental properties. Upon increasing demand on the device applications, graphene is viewed as a prototypical system for engineering $v_{\mathrm{F}}$. Indeed, several efforts have succeeded in modifying $v_{\mathrm{F}}$ by varying charge carrier concentration, $n$. Here we present a powerful but simple new way to engineer $v_{\mathrm{F}}$ while holding $n$ constant. We find that when the environment embedding graphene is modified, the $v_{\mathrm{F}}$ of graphene is (i) inversely proportional to its dielectric constant, reaching $v_{\mathrm{F}} \sim 2.5 \times 10^{6} \mathrm{~m} / \mathrm{s}$, the highest value for graphene on any substrate studied so far and (ii) clearly distinguished from an ordinary Fermi liquid. The method demonstrated here provides a new route toward Fermi velocity engineering in a variety of two-dimensional electron systems including topological insulators.
\end{abstract}

D

ue to its lattice structure and position of the Fermi energy, the low-energy electronic excitations of graphene are described by an effective field theory that is Lorentz invariant ${ }^{1}$. Unlike Galilean invariant theories such as Fermi Liquids ${ }^{2}$ whose main relevant parameter is the effective mass, Lorentz invariant theories are characterized by an effective velocity. Because of this, an increase of electron-electron interactions induces an increase of the Fermi velocity, $v_{\mathrm{F}}$, in contrast to Fermi liquids, where the opposite trend is true ${ }^{3}$. In the case of graphene, when electron-electron interactions are weak ${ }^{4}, v_{\mathrm{F}}$ is expected to be as low as $0.85 \times 10^{6} \mathrm{~m} / \mathrm{s}$, whereas, for the case of strong interactions ${ }^{5}, v_{\mathrm{F}}$ is expected to be as high as $1.73 \times 10^{6} \mathrm{~m} / \mathrm{s}$.

Recently, Fermi velocities as high as $\sim 3 \times 10^{6} \mathrm{~m} / \mathrm{s}^{6}$ have been achieved in suspended graphene through a change of the carrier concentration $n^{6-9}$. However, because this dependence is logarithmic, $n$ needs to be changed by two orders of magnitude in order to change the velocity by a factor of 3 . This implies that it is unpractical to use $n$ as a way to engineer $v_{\mathrm{F}}$, let alone the fact that one should first realize suspended graphene in the device ${ }^{6}$. Several other routes have also been proposed to engineer $v_{\mathrm{F}}$ in graphene via the electron-electron interaction, including modifications of: a) curvature of the graphene sheet ${ }^{10}$; b) periodic potentials ${ }^{11}$; ) dielectric screening ${ }^{12-14}$. While the former two also substantially modify the starting material, the latter simply modifies the effective dielectric constant, $\varepsilon$, making it more appealing for device applications ${ }^{15}$. Despite this advantage, no systematic study of how to engineer $v_{\mathrm{F}}$ by changing $\varepsilon$ exists to date. Here we provide a new venue to control the Fermi velocity of graphene using dielectrics, while keeping $n$ constant.

\section{Results}

We perform such a study using three single-layer graphene samples, which were prepared by chemical vapor deposition (CVD) on $\mathrm{Cu}$, followed by an in situ dewetting of $\mathrm{Cu}$ on quartz (single crystal $\left.\mathrm{SiO}_{2}\right)^{16}$ or a transfer onto hexagonal boron nitride $(\mathrm{BN})^{17}$, and by epitaxial growth on $4 H-\mathrm{SiC}(000-1)^{18}$. Figures $1 \mathrm{~A}$ and $1 \mathrm{~B}$ show angleresolved photoemission spectroscopy (ARPES) intensity maps measured near the Brillouin zone corner $\mathrm{K}$ along the $\Gamma-\mathrm{K}$ direction for the two CVD grown samples, which constitute the first report on Dirac quasiparticle mapping from these samples. Following the maximum intensity, one can clearly observe almost linear energy spectra, characteristic of Dirac electrons ${ }^{19}$. The momentum distribution curves (MDC), intensity spectra taken at constant energy as a function of momentum, are shown in Fig. 1C. In addition to being proportional to the imaginary part of the electron self-energy, the MDC spectral width provides information on the sample quality. A clear increase of the width is observed by changing the substrate from $\mathrm{SiC}(000-1)$ via $\mathrm{BN}$ to quartz, a trend that is in overall agreement with the theoretical expectation that the electron self-energy should vary with the inverse square of the dielectric screening ${ }^{20}$, as later discussed. The quartz sample here used constitutes a substantial 


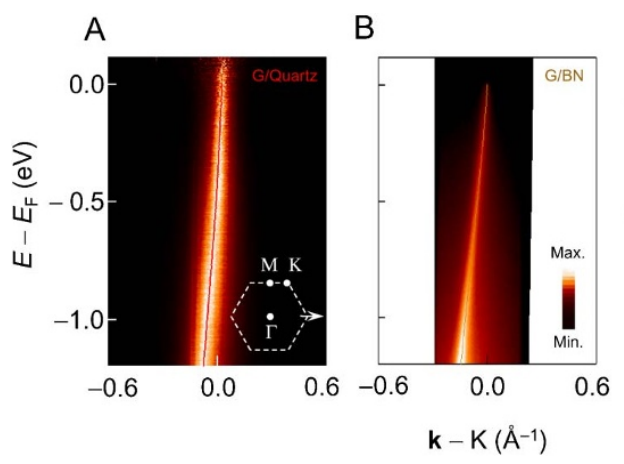

C

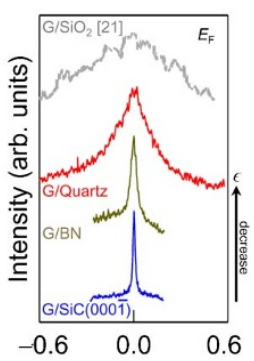

Figure $1 \mid$ ARPES intensity maps of graphene on quartz and BN. (A-B) Normalized and raw ARPES intensity maps of graphene/quartz (panel (A)) and graphene/BN (panel (B)), respectively. The red and darkyellow lines are the dispersions, obtained by fitting momentum distribution curves (MDCs). (C) MDCs at $E_{\mathrm{F}}$ for graphene on $\mathrm{SiC}(000-1)$ (blue line), $\mathrm{BN}$ (dark-yellow line), quartz (red line), and $\mathrm{SiO}_{2}{ }^{21}$ (gray-dashed line).

improvement over a previous experiment on a similar substrate ${ }^{21}$ (compare $0.19 \AA^{-1}$ (red line) versus $\sim 0.7 \AA^{-1}$ (gray-dashed line)). The much improved data quality allows for a detailed self-energy analysis and consequent extraction of important parameters such as $v_{\mathrm{F}}$.

To understand how the dielectric substrate affects the electronic properties, in Fig. 2, we show the energy vs. momentum dispersions for graphene on three different substrates, $\mathrm{SiC}(000-1), \mathrm{BN}$, and quartz, obtained by fitting the MDC spectra. The observed dispersions exhibit two distinctive features. First, the measured dispersions deviate from linearity with an increased slope around $\sim 0.5 \mathrm{eV}$ for all the samples (compare experimental data to dashed gray lines in Fig. 2A). As the substrate is changed from $\mathrm{SiC}(000-1)$ via $\mathrm{BN}$ to quartz, corresponding to a decrease of the dielectric screening, the departure from linearity at high energy becomes more pronounced. Second, the direct comparison between experimental dispersions and $a b$ initio calculations for the two extreme cases $\varepsilon=1^{5}$ (suspended graphene) and $\varepsilon=\infty^{4}$ shows another substrate-dependence (Fig. 2B). Upon changing the substrate, the slope increases approaching the dispersion for $\varepsilon=1$. The deviation from linearity and the enhancement of the slope result in a reshape of the typical conical dispersion, in a similar fashion as reported for other charge-neutral graphene samples $^{6,12}$ (see cartoons in the inset of Fig. 2A: from left to right). We note that the largest upturn for graphene/quartz cannot be explained
A

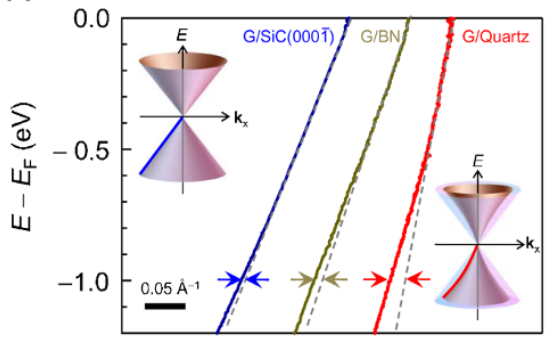

k
B

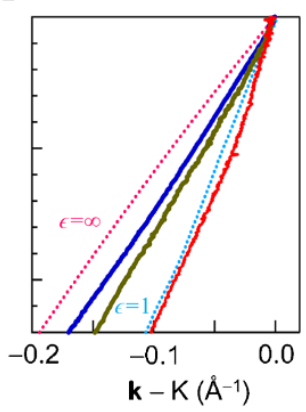

Figure 2 Experimental and theoretical energy spectra for different dielectric constants. (A) Experimental dispersions for graphene on $\mathrm{SiC}(000-1)$ (blue line), BN (dark-yellow line), and quartz (red line). The gray-dashed lines are guides to the eyes. The insets are cartoons for the electron band structure of graphene with weak (left) and strong (right) electron-electron interactions. The data are shifted along the $x$-axis. (B) The direct comparison of experimental dispersions with theories:

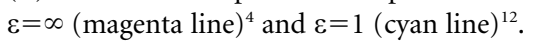

by: a) resolution, which typically results in the deflection of MDC peaks near $E_{\mathrm{F}}$ to lower momentum, and would involve a much smaller effect by an order of magnitude $(\leq \mathrm{a} \text { few tens } \mathrm{meV})^{22}$; b) the presence of other bands with a different azimuthal orientation, which would cause instead an abrupt increase and a significant asymmetry of the MDC width at the upturn energy.

\section{Discussion}

To quantify the effect of dielectric substrates on the electron-electron interactions and $v_{\mathrm{F}}$, we adopt the standard self-energy analysis to extract self-consistently the strength of the electron-electron interactions and $\varepsilon^{1,12,23,24}$. Figure $3 \mathrm{~A}$ shows the difference between measured dispersions, $E(\mathbf{k})$ (from Fig. 2A), and the theoretical dispersion for $\varepsilon=\infty, E_{\mathrm{LDA}}(\mathbf{k})$ (shown in Fig. 2B). Assuming that electron-electron interactions are effectively screened for $\varepsilon=\infty$, the $E$ - $E_{\mathrm{LDA}}$ curve can be considered a good measurement of the difference between the self-energy and its value at $E_{\mathrm{F}}$. To fit these curves, we use the marginal Fermi liquid self-energy function as previously reported ${ }^{12,23}$ with an analytic form of $\alpha \hbar v_{0} / 4 \times\left(\mathbf{k}-\mathbf{k}_{\mathrm{F}}\right) \ln \left(\mathbf{k}_{\mathrm{C}} /\left(\mathbf{k}-\mathbf{k}_{\mathrm{F}}\right)\right.$ ) (dotted lines in Fig. 3A). Here, $\alpha$ is a dimensionless fine-structure constant (or the strength of electron-electron interactions) defined as $e^{2} /\left(4 \pi \varepsilon \hbar v_{0}\right)^{23}, v_{0}$ the Fermi velocity for $\varepsilon=\infty, 0.85 \times 10^{6} \mathrm{~m} / \mathrm{s}^{4}, \mathbf{k}_{\mathrm{C}}$ the momentum cutoff, $1.7 \AA^{-1}$, and $\mathrm{k}_{\mathrm{F}}$ the Fermi wave number. An overall good agreement with the experimental data is observed allowing us to extract important parameters such as $\varepsilon$ and $\alpha$ for graphene on each substrate. For graphene on $\mathrm{SiC}(000-1)$ and $\mathrm{BN}$, we obtain $\varepsilon=7.26 \pm 0.02$ $(\alpha=0.35)$ and $\varepsilon=4.22 \pm 0.01(\alpha=0.61)$, respectively. The extracted value for graphene on $\mathrm{BN}$ is in agreement with the standard approximation $\varepsilon=\left(\varepsilon_{\text {vacuum }}+\varepsilon_{\text {substrate }}\right) / 2=4.02$ and 3.05 , where $\varepsilon_{\text {vacuum }}=1$ and $\varepsilon_{\text {substrate }}=7.04$ (for out-of-plane polarization) and 5.09 (for inplane polarization) in the low frequency limit (static dielectric constant) for hexagonal-BN ${ }^{25}$. Similarly, the obtained value for graphene on $\mathrm{SiC}(000-1)$ is close to a previous report ${ }^{12}$. The apparent discrepancy with the latter (compare $\varepsilon=7.26 \pm 0.02$ in this work with $6.4 \pm 0.1$ in reference ${ }^{12}$ ) is due to the different choice of reference band (or so-called bare band). Specifically, in this work, $E_{\mathrm{LDA}}$ is used as the bare band, whereas, in reference 12 , the bare band is approximated by a straight line. Finally, for graphene/quartz, we obtain $\varepsilon=1.80 \pm 0.02(\alpha=1.43)$, which is smaller than the expected value of $\varepsilon=2.45^{26}$, instead closer to the experimentally extracted value for suspended graphene $(\sim 2.2)^{6}$. This observation, together with the similar energy-momentum dispersion relation at high binding energy to the theoretical one for suspended graphene (Fig. 2B), points to a very weak effect of the substrate. This is likely a consequence of the different sample preparation method adopted here (see Methods section).

In Fig. 3B, we show the measured $v_{\mathrm{F}}$ as a function of the extracted $\varepsilon$ (see also Table 1). Results from a suspended sample ${ }^{6}$ and another graphene $/ \mathrm{SiO}_{2}$ sample ${ }^{21}$ are also plotted for comparison. Upon decreasing $\varepsilon$ from $\infty$ to 7.26 and $4.22, v_{\mathrm{F}}$ is enhanced from its LDA limit of $0.85 \times 10^{6} \mathrm{~m} / \mathrm{s}$ (cyan triangle in Fig. $3 \mathrm{~B}$ ) to $1.15 \pm 0.02 \times 10^{6} \mathrm{~m} / \mathrm{s}$ (blue circle in Fig. $3 \mathrm{~B}$ ) and $1.49 \pm 0.08 \times 10^{6} \mathrm{~m} / \mathrm{s}$ (dark-yellow circle in Fig. $3 \mathrm{~B}$ ), by $35 \%$ and $75 \%$, respectively. Surprisingly, when $\varepsilon$ is further decreased to 1.80 , a dramatic enhancement of $v_{\mathrm{F}}$ up to $2.49 \pm 0.30 \times 10^{6} \mathrm{~m} / \mathrm{s}$ (red circle in Fig. 3B) is observed. Such enhancement corresponds to a $190 \%$ increase from its bare value and represents the highest value reported for graphene on any substrate $^{27-29}$. Interestingly, this velocity is comparable to the value measured for suspended graphene (green square in Fig. 3B) ${ }^{6}$. Clearly, a $1 / \varepsilon$ dependence of $v_{F}$ is observed (dashed line in Fig. 3B) in agreement with the theoretical prediction ${ }^{6,23}$. Our result constitutes the first observation of a power law dependence of the Fermi velocity on the dielectric constant at fixed $n$. This power law dependence allows one to achieve, by a smart choice of dielectric, a high value of $v_{\mathrm{F}}$ that cannot be attained otherwise by changing $n^{6}$. 
A

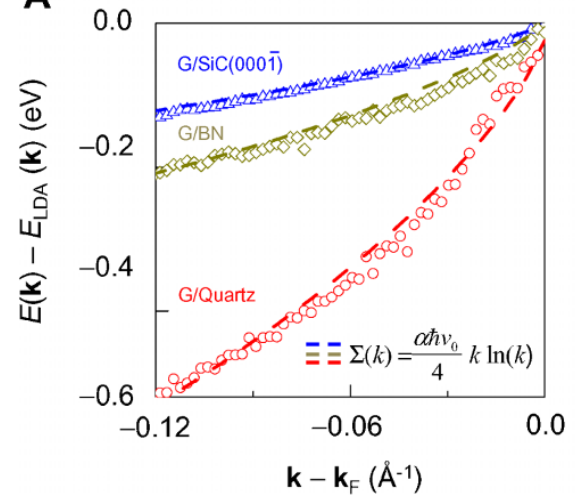

B

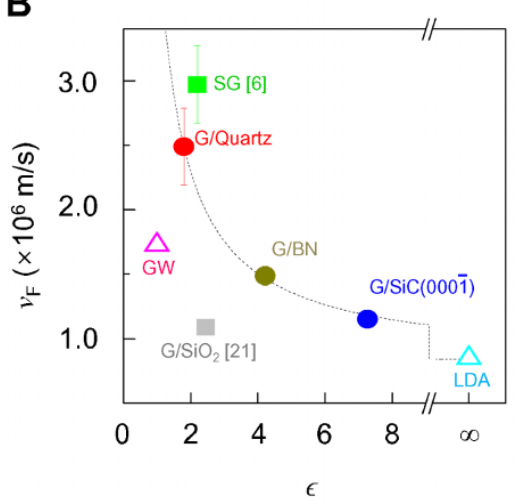

C

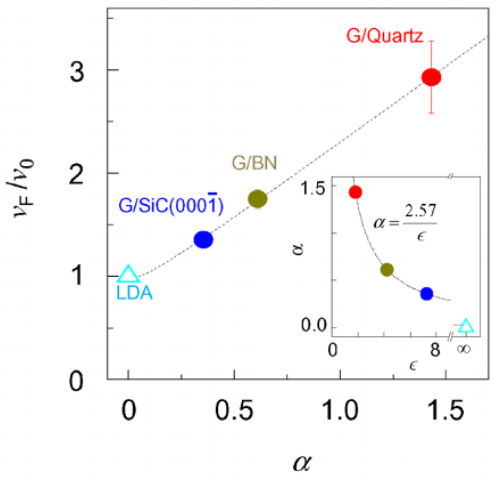

Figure $3 \mid$ Fermi velocity and the strength of electron-electron interactions. (A) $E$ - $E_{\mathrm{LDA}}$ dispersions for graphene on $\mathrm{SiC}(000-1)(\mathrm{blue}$ line), $\mathrm{BN}$ (darkyellow line), and quartz (red line). (B) Fermi velocities as a function of $\varepsilon$. The dashed line is a theoretical curve for $v_{\mathrm{F}}$, which is inversely proportional to $\varepsilon^{6,23}$. Filled symbols correspond to experimental results, while empty symbols to theoretical values. $\varepsilon=2.45$ for $\mathrm{G} / \mathrm{SiO}_{2}{ }^{26}$ is obtained from the standard approximation, $\varepsilon=\left(\varepsilon_{\text {vacuum }}+\varepsilon_{\text {substrate }}\right) / 2$ (see text). (C) The ratio of $v_{\mathrm{F}}$, the renormalized Fermi velocity due to electron-electron interactions, to $v_{0}=0.85 \times 10^{6} \mathrm{~m} / \mathrm{s}$, the bare Fermi velocity in the LDA limit where $\varepsilon=\infty^{4}$, as a function of $\alpha$. The dashed line is the fit given by $v_{\mathrm{F}} / v_{0}=1-3.28 \alpha\{1+(1 / 4)$ $\ln [(1+4 \alpha) / 4 \alpha]-1.45\}^{31}$ for charge neutral graphene. The inset is the relation between $\alpha$ and $\varepsilon$, where the dashed line is a $\alpha=e^{2} /\left(4 \pi \varepsilon \hbar v_{0}\right)=2.57 / \varepsilon$ curve $e^{23}$.

We note that CVD graphene on quartz (red circle in Fig. 3B) exhibits higher $v_{\mathrm{F}}$ than exfoliated graphene on amorphous $\mathrm{SiO}_{2}$ (gray square in Fig. 3B) with the same stoichiometry as quartz. This is a consequence of different sample preparation process and is due to the larger presence of impurities in the exfoliated sample, as suggested by the extremely broad spectra (see gray dashed line in Fig. 1C). Therefore, although, in theory, one should expect smaller $\nu_{\mathrm{F}}$ due to screened electron-electron interactions from impurity ${ }^{13}$, one should be cautious in extracting meaningful parameters from these data. We also note that $a b$ initio $G W$ calculations $^{5}$ (magenta triangle in Fig. $3 \mathrm{~B}$ ) underestimate $v_{\mathrm{F}}$ of suspended graphene. This may be due to the finite $k$-point sampling inherent in such calculations, or it could also be an indication of the need to add higher-order terms in the self-energy calculation by the $G W$-approximation.

In Fig. 3C, we plot the ratio between $v_{\mathrm{F}}$ and $v_{0}$, the expected Fermi velocity in the fully screened case $(\varepsilon=\infty)$, as a function of $\alpha$. As the strength of electron-electron interactions is increased, $v_{\mathrm{F}}$ is also enhanced. This is in striking difference with the standard Fermi liquid picture, where $v_{\mathrm{F}}$ is expected to decrease with increasing $\alpha^{30}$. On the other hand, the observed behavior is consistent with previous theoretical studies for graphene in the case of specific electron-electron interactions $s^{30,31}$ (dashed line in Fig. 3C) exhibiting the characteristic self-energy spectrum analogous to a marginal Fermi liquid ${ }^{1}$. As a result, the departure from the Fermi liquid picture becomes more important with increasing electron-electron interactions or decreasing dielectric screening (see the relation between $\alpha$ and $\varepsilon$ in the inset of Fig. 3C). Additionally, the observation of $\alpha$ value close to 1 (neither $\alpha \ll 1$ nor $\alpha \gg 1$ ) for graphene/quartz may indicate that a full theoretical treatment beyond the random-phase approximation ${ }^{1}$ may be required to understand this sample and/or suspended graphene ${ }^{6}$.

The very good agreement with theoretical prediction $\mathrm{s}^{23,31}$ for both $v_{\mathrm{F}}$ versus $\varepsilon$ (Fig. 3B) and $v_{\mathrm{F}}$ versus $\alpha$ (Fig. 3C) confirms that the dielectric constants obtained by the self-energy analysis are self-consistent. Finally the experimentally determined $\varepsilon$ can largely account

Table 1 | Fermi velocity $\left(v_{\mathrm{F}}\right)$, dielectric constant $(\varepsilon)$, and fine structure constant $(\alpha)$ of graphene on each substrate

\begin{tabular}{lccc} 
Substrate & $v_{\mathrm{F}} \times 10^{6} \mathrm{~m} / \mathrm{s}$ & $\varepsilon$ & $\alpha$ \\
\hline Metals (LDA) & 0.85 & $\infty$ & - \\
$\mathrm{SiC}(000-1)$ & $1.15 \pm 0.02$ & $7.26 \pm 0.02$ & 0.35 \\
$h-\mathrm{BN}$ & $1.49 \pm 0.08$ & $4.22 \pm 0.01$ & 0.61 \\
Quartz & $2.49 \pm 0.30$ & $1.80 \pm 0.02$ & 1.43 \\
\hline
\end{tabular}

for the relatively broad MDCs observed for graphene on quartz (Fig. 1C), as compared to graphene on $\mathrm{BN}$ and $\mathrm{SiC}(000-1)$. For $\varepsilon$ values of $1.80,4.22$, and 7.26 , for graphene on quartz, $\mathrm{BN}$, and $\mathrm{SiC}(000-1)$ respectively, the MDC widths, expected to vary with the inverse square of the dielectric screening ${ }^{20}$, should be roughly 16 and 5 times broader for graphene on quartz and BN than graphene on $\mathrm{SiC}(000-1)$, in line with the experimental observation (see, for example, Fig. 1C). We stress that, contrary to a Fermi liquid system, the broader MDC spectra observed for graphene/quartz do not necessarily imply decreased transport properties. On the contrary, the enhanced $\alpha$, the primary cause of the broad spectra, give rise to an enhancement of Fermi velocity, which is ultimately one of the most important parameters for device applications.

In conclusion, we have unveiled the crucial role of dielectric screening in graphene to control both Fermi velocity and electronelectron interactions. Additionally, we have shown that graphene, in its charge neutral state, departs from a standard Fermi liquid not only in its logarithmic energy spectrum as previously discussed ${ }^{12}$, but also in the way that $v_{\mathrm{F}}$ is modulated by the strength of electron-electron interactions. This dependence provides an alternative way to engineer Fermi velocity for graphene on a substrate by modifying the dielectric substrate. This approach can also be applied to chargedoped graphene and other two-dimensional electron systems such as topological insulators ${ }^{32}$ that can be grown or transferred to dielectric substrates.

\section{Methods}

Graphene samples were prepared in three different ways: epitaxial growth on the surface of a $4 H$-SiC(000-1) substrate; chemical vapor deposition (CVD) growth on a $\mathrm{Cu}$ film followed by a transfer onto the surface of boron nitride ${ }^{17}$; and CVD growth followed by in situ dewetting of Cu layer in between graphene and a single crystal $\mathrm{SiO}_{2}$ (namely quartz which is different from amorphous $\mathrm{SiO}_{2}$ on an $\mathrm{Si}$ substrate, the widely used substrate for exfoliated graphene ${ }^{27}$ ) substrate ${ }^{16}$. The later procedure is clearly different from the standard method of exfoliating graphite followed by deposition onto the amorphous $\mathrm{SiO}_{2}$ layer ${ }^{21}$. This results in a reduced effect of the substrate that is suggested by the enhanced height variation with respect to the substrate compared to the sample prepared by the exfoliation and deposition ${ }^{16,33}$. The resulting graphene is more decoupled from the substrate as supported by several features such as Fermi velocity, dielectric constant, and the electron band at higher energies closer to suspended sample.

In order to remove any residue including $\mathrm{Cu}$ and $\mathrm{PMMA}$, a precursor to grow $\mathrm{CVD}$ graphene and a polymer to transfer graphene, respectively, we heated the sample to $1000{ }^{\circ} \mathrm{C}$ in ultra-high vacuum. The removal of $\mathrm{Cu}$ is confirmed by: (a) optical microscopy showing a cleaner image without residual $\mathrm{Cu}$ once the sample has been heated; (b) absence of related $\mathrm{Cu}$ features in the ARPES spectra such as $3 d$ electrons at $3.0 \mathrm{eV}$ and $3.5 \mathrm{eV}$ below Fermi energy, and $4 s$ free-electron-like state with a band minimum at $0.25 \mathrm{eV}$ below Fermi energy ${ }^{34}$. 
High-resolution ARPES experiments have been performed at beamline 10.0.1.1 of the Advanced Light Source at Lawrence Berkeley National Laboratory using $50 \mathrm{eV}$ photons at $15 \mathrm{~K}$. Energy and angular (momentum) resolutions were set to be $22 \mathrm{meV}$ and $0.2^{\circ}\left(\sim 0.01 \AA^{-1}\right)$, respectively.

1. Kotov, V. N., Uchoa, B., Pereira, V. M., Castro Neto, A. H. \& Guinea, F. ElectronElectron Interactions in Graphene: Current Status and Perspectives. Rev. Mod. Phys. 84, 1067 (2012).

2. Landau, L. Theory of Fermi-liquids. Soviet Physics JEPT 3, 920 (1957)

3. Ashcroft, N. W. \& Mermin, N. D. Solid State Physics (Saunders College, New York, 1976).

4. Trevisanutto, P. E., Giorgetti, C., Reining, L., Ladisa, M. \& Olevano, V. Ab Initio GW Many-Body Effects in Graphene.Phys. Rev. Lett. 101, 226405 (2008).

5. Park, C.-H., Giustino, F., Spataru, C. D., Cohen, M. L. \& Louie, S. G. AngleResolved Photoemission Spectra of Graphene from First-Principles Calculations. Nano Lett. 9, 4234-4239 (2009).

6. Elias, D. C. et al. Dirac cones reshaped by interaction effects in suspended graphene. Nat. Phys. 7, 701-704 (2011).

7. Bostwick, A. et al. Renormalization of graphene bands by many-body interactions. Solid State Commun. 143, 63-71 (2007).

8. Li, Z. Q. et al. Dirac charge dynamics in graphene by infrared spectroscopy. Nat. Phys. 4, 532-535 (2008).

9. Li, G., Luican, A. \& Andrei, E. Y. Scanning Tunneling Spectroscopy of Graphene on Graphite. Phys. Rev. Lett. 102, 176804 (2009).

10. Du, X., Skachko, I., Barker, A. \& Andrei, E. Y. Approaching ballistic transport in suspended graphene. Nat. Nano. 3, 491-495 (2008).

11. Park, C.-H., Yang, L., Son, Y.-W., Cohen, M. L. \& Louie, S. G. Anisotropic behaviors of massless Dirac fermions in graphene under periodic potentials. Nat. Phys. 4, 213-217 (2008).

12. Siegel, D. A. et al. Many-body interactions in quasi-freestanding graphene. Proc. Natl. Acad. Sci. USA 108, 11365-11369 (2011).

13. Jang, C. et al. Tuning the Effective Fine Structure Constant in Graphene: Opposing Effects of Dielectric Screening on Short- and Long-Range Potential Scattering. Phys. Rev. Lett. 101, 146805 (2008).

14. Raoux, A. et al. Velocity-modulation control of electron-wave propagation in graphene. Phys. Rev. B 81, 073407 (2010).

15. Castro Neto, A. H. \& Novoselov, K. New directions in science and technology: two-dimensional crystals. Rep. Prog. Phys. 74, 082501 (2011).

16. Ismach, A. et al. Direct Chemical Vapor Deposition of Graphene on Dielectric Surfaces. Nano Lett. 10, 1542-1548 (2010).

17. Dean, C. R. et al. Boron nitride substrates for high-quality graphene electronics. Nat. Nano. 5, 722-726 (2010).

18. Hass, J. et al. Structural properties of the multilayer graphene/4H-SiC $(000-1)$ system as determined by surface x-ray diffraction. Phys. Rev. B 75, 214109 (2007).

19. Zhou, S. Y. et al. Substrate-induced bandgap opening in epitaxial graphene. Nat. Mater. 6, 770-775 (2007).

20. González, J., Guinea, F. \& Vozmediano, M. A. H. Unconventional Quasiparticle Lifetime in Graphite. Phys. Rev. Lett. 77, 3589-3592 (1996).

21. Knox, K. R. et al. Spectromiscroscopy of single and multilayer graphene supported by a weakly interacting substrate. Phys. Rev. B 78, 201408(R) (2008).
22. Plumb, N. C. et al. Low-Energy $(<10 \mathrm{meV})$ Feature in the Nodal Electron SelfEnergy and Strong Temperature Dependence of the Fermi Velocity in $\mathrm{Bi}_{2} \mathrm{Sr}_{2} \mathrm{CaCu}_{2} \mathrm{O}_{8+\delta}$. Phys. Rev. Lett. 105, 046402 (2010).

23. González, J., Guinea, F. \& Vozmediano, M. A. H. Non-Fermi liquid behavior of electrons in the half-filled honeycomb lattice (A renormalization group approach). Nucl. Phys. B 424, 595-618 (1994).

24. Damascelli, A., Hussain, Z. \& Shen, Z. X. Angle-resolved photoemission studies of the cuprate superconductors. Rev. Mod. Phys. 75, 473-541 (2003).

25. Geick, R., Perry, C. H. \& Rupprecht, G. Normal Modes in Hexagonal Boron Nitride. Phys. Rev. 146, 543-547 (1966).

26. Gray, P. R. et al. Analysis and Design of Analog Integrated Circuits (Wiley, New York, 1984).

27. Novoselov, K. S. et al. Two-dimensional gas of massless Dirac fermions in graphene. Nature 438, 197-200 (2005).

28. Berger, C. et al. Electronic Confinement and Coherence in Patterned Epitaxial Graphene. Science 312, 1191-1196 (2006).

29. Sprinkle, M. et al. First Direct Observation of a Nearly Ideal Graphene Band Structure. Phys. Rev. Lett. 103, 226803 (2009).

30. Polini, M., Asgari, R., Barlas, Y., Pereg-Barnea, T. \& MacDonald, A. H. Graphene: A pseudochiral Fermi liquid. Solid State Commun. 143, 58-62 (2007).

31. Das Sarma, S., Hwang, E. H. \& Tse, W.-K. Many-body interaction effects in doped and undoped graphene: Fermi liquid versus non-Fermi liquid. Phys. Rev. B 75, 121406(R) (2007).

32. Hsieh, D. et al. Observation of Unconventional Quantum Spin Textures in Topological Insulators. Science 323, 919-922 (2009).

33. Yeh, N.-C. et al. Scanning Tunneling Spectroscopic Studies of the Effects of Dielectrics and Metallic Substrates on the Local Electronic Characteristics of Graphene. ECS Transactions 28, 115-123 (2010).

34. Patthey, F., Schaffner, M.-H., Schneider, W.-D. \& Delley, B. Observation of a Fano Resonance in Photoemission. Phys. Rev. Lett. 82, 2971-2974 (1999).

\section{Acknowledgements}

This work was supported by the Director, Office of Science, Office of Basic Energy Sciences, Materials Sciences and Engineering Division, of the U.S. Department of Energy under Contract No. DE-AC02-05CH11231.

\section{Author contribution}

A.L. and C.G.H. designed the experiments and C.G.H., D.A.S., and S.-K.M. carried them out. C.G.H., W.R., A.I., Y.Z., and A.Z. prepared graphene samples. Everyone contributed to the writing of the paper.

\section{Additional information}

Competing financial interests: The authors declare no competing financial interests.

License: This work is licensed under a Creative Commons

Attribution-NonCommercial-NoDerivative Works 3.0 Unported License. To view a copy of this license, visit http://creativecommons.org/licenses/by-nc-nd/3.0/

How to cite this article: Hwang, C. et al. Fermi velocity engineering in graphene by substrate modification. Sci. Rep. 2, 590; DOI:10.1038/srep00590 (2012). 